\title{
ASSISTÊNCIA DOMICILIARIA AO IDOSO: PERFIL DO CUIDADOR FORMAL - PARTE II*
}

\author{
THE HOME CARE OF ELDERLY: CAREGIVER PROFILE - PART TWO
}

\author{
ASISTENCIA DOMICILIAR AL ANCIANO: \\ PERFIL DEL CUIDADOR FORMAL - PARTE II
}

\author{
Kozue Kawasaki** \\ Maria José D'Elboux Diogo***
}

Kawasaki K, Diogo MJD. Assistência domiciliária ao idoso: perfil do cuidador formal - parte II. Rev Esc Enferm USP 2001; 35(4): 320-7.

\section{RESUMO}

O idoso fragilizado, mantido em seu domicílio, requer cuidados específicos, os quais são realizados muitas vezes por pessoas contratadas pela família para tal, denominadas cuidadores formais. Frente o aumento da oferta de trabalho destas pessoas e a escassez de literatura nacional sobre seu perfiL, desenvolvemos um estudo com 41 pessoas que ofereceram seus serviços através de anúncios em dois jornais de maior circulação no município de Campinas, São Paulo. Tal estudo teve por objetivos: 1. caracterizar estes cuidadores (este objetivo foi contemplado com um artigo publicado anteriormente) e 2. verificar as atividades propostas para a assistência ao idoso. No presente trabalho serão apresentados os dados referentes ao segundo objetivo.

PALAVRAS -CHAVE: Idosos. Cuidados domiciliares de saúde. Cuidadores.

\begin{abstract}
The frail elderly, maintained in its home, request specific cares, which are accomplished many times by people contracted, denominated formal caregivers. With the increase of the offer of these people's work and the shortage literature on its profile, we developed a study with 41 advertisers that offered their services in two newspapers of larger circulation in the city of Campinas, São Paulo, with the following objectives: 1. to characterize these caregivers and 2. to verify the activities proposals for care to the elderly. In the present work the referring data are presented to the second objective.
\end{abstract}

KEYWORDS: Elderly. Home care. Caregivers.

\section{RESUMEN}

El anciano debilitado, mantenido en su habitación, carece de cuidados específicos, los cuales han sido realizados muchas veces por personas contratadas, denominadas cuidadores formales. Frente al aumento de oferta de trabajo de estas personas y a la escasez de literatura sobre el perfil, desarrollamos un estudio con 41 anunciantes que ofrecieron sus servicios dos periódicos de grande circulación en el municipio de Campinas, San Pablo, con los siguientes objetivos: 1. caracterizar estos cuidadores y 2 . verificar las actividades propuestas para la asistencial al anciano. El presente trabajo presenta los datos referentes a lo secundo objetivo.

PALABRAS-CLAVE: Anciano. Cuidados domiciliários de salud. Cuidadores.

Parte do trabalho de Iniciação científica com financiamento da FAPESP(Proc. $N^{\circ}$ 97/13702-9). Tema livre apresentado no $51^{\circ}$ Congresso Brasileiro de Enfermagem, de 02 a 07 de outubro de 1999, Florianópolis, SC.

** Graduanda do Curso de Graduação em Enfermagem da Faculdade de Ciências Médicas da UNICAMP.

*** Enfermeira. Professor Assistente Doutor no Departamento de Enfermagem da Faculdade de Ciências Médicas da Universidade Estadual de Campinas. Email: mariadio@uol.com.br 


\section{INTRODUÇÃO}

O atendimento ou cuidado domiciliário ao idoso é um segmento da área de saúde cuja tendência é se expandir rapidamente. A residência do paciente é um campo novo de trabalho para diversos profissionais e uma alternativa para muitas pessoas com baixa qualificação, que frente as dificuldades advindas da falta de emprego na nossa realidade, buscam novos espaços, entre eles, o trabalho remunerado em domicílios. Estas pessoas oferecem seus serviços sob formas variadas em jornais, revistas, tablóides, como por exemplo: "acompanhante de idosos...", "dama de companhia para senhoras...", "cuido de idosos...", ".. com prática para cuidar de idosos", entre Outros.

Os cuidadores formais, assim denominados, conforme detalhamento na Parte I deste estudo, têm ocupado um espaço significativo no cuidado do idoso fragilizado ou dependente, no seu domicílio. Entre as diferentes atividades a serem realizadas por esses indivíduos destaca-se a assistência ao idoso, cuja abrangência não é muito clara.

Esta realidade tem despertado intensa preocupação e muitas discussões entre os profissionais de enfermagem em geral e na área de gerontologia especificamente. Vale destacar o posicionamento do Conselho Regional de Enfermagem de São Paulo quanto aos cursos de "cuidadores de idoso"('

Não pretendemos neste momento discorrer sobre a formação de recursos humanos em gerontologia e em particular dos cuidadores. Na realidade, está havendo a inserção destes cuidadores no mercado de trabalho, que com ou sem competência, estão assumindo a assistência do idoso no domicílio.

Frente a escassez de literatura científica publicada sobre esta questão e o aumento da oferta dos serviços dessas pessoas principalmente na imprensa escrita, é imprescindível conhecermos as características dos indivíduos que dispensam cuidados aos idosos no domicílio.

\section{OBJETIVO}

Temos como objetivo, verificar as atividades propostas pelas pessoas que se oferecem para cuidar de idosos no domicilio, no município de Campinas, São Paulo.

\section{SUJEITOS E MÉTODO}

Trata-se de um estudo exploratório realizado com 41 pessoas, de ambos os sexos que ofereceram. seus serviços para cuidar de idosos no domicílio, em anúncios em dois jornais de maior circulação do municipio de Campinas, estado de São Paulo.

Realizamos a entrevista por meio do contato telefônico, e informamos o entrevistado quanto aos objetivos da pesquisa, garantindo o seu anonimato e respeitando a não concordância do mesmo em participar deste trabalho. Com a finalidade de homogeneizar a necessidade dos cuidados a serem dispensados pelo cuidador, foi apresentado a ele, uma situação hipotética, assim descrita: "um idoso de 83 anos, acamado, com sequela de derrame cerebral o qual deixou seu lado esquerdo paralisado. E dependente, necessitando de auxílio para a alimentação, higiene, sendo sua micção e evacuação realizados no leito. Apresenta ainda dificuldade na comunicação pois não escuta bem. Mora com sua esposa, também idosa $(80$ anos), sem condições de prestar cuidados ao marido."

No presente trabalho serão apresentados os resultados obtidos da segunda parte do instrumento utilizado para a coleta de dados na pesquisa como um todo. Esta parte lista as atividades propostas para a assistência ao idoso, que foram subdivididas em cuidados de enfermagem e atividades instrumentais da vida diária (AIVDs). Os cuidados de enfermagem relacionam-se basicamente as atividades da vida diária (AVDs): a alimentação, as eliminações, a terapêutica, a integridade cutânea, a higiene, a locomoção e a movimentação. As AIVDs são as atividades que subsidiam a vida cotidiana e requerem melhor desempenho da capacidade funcional, portanto, no idoso fragilizado ou com limitação da sua capacidade funcional, são as primeiras a serem comprometidas. Para este estudo serão consideradas as seguintes AIVDs: lavar e passar as roupas, preparar as refeições, limpar a casa e atividades extradomiciliares (ir ao banco, padaria, farmácia, supermercado, etc.).

Quanto aos cuidados de enfermagem referentes a alimentação, os itens administração da dieta e os cuidados com a jejunostomia foram separados das demais vias enterais devido suas peculiaridades na assistência.

Cabe ressaltar que o termo qualificação foi utilizado para designar as diferentes categorias dos anunciantes, mesmo considerando que o acompanhante e o atendente de enfermagem não possuam qualificação específica.

Os dados serão apresentados em tabelas onde foram empregadas as abreviações para as qualificações: E para o enfermeiro, TE para o técnico, $\mathrm{AE}$ ao auxiliar, AT para o atendente e AC para o acompanhante. 


\section{RESULTADOS E DISCUSSÃO}

Os resultados serão apresentados e discutidos conforme o roteiro do instrumento utilizado para a coleta de dados (ANEXO I). Por tratar-se de um estudo exploratório descritivo, no qual o objetivo foi verificar as atividades realizadas pelos cuidadores formais, a discussão será restrita aos dados e a nossa experiência profissional, uma vez que no levantamento bibliográfico realizado não encontramos outros trabalhos desta natureza, o que impediu o diálogo com outros autores.
Vale lembrar que foram entrevistados três enfermeiros, uma técnica de enfermagem, dez auxiliares de enfermagem, cinco atendentes de enfermagem e 22 acompanhantes.

\section{Cuidados de Enfermagem}

Para cuidados de enfermagem abordados neste trabalho não nos deteremos ao detalhamento técnico específico de cada procedimento. Outrossim, convém ressaltar que a assistência ao idoso compreende muito além das atividades investigadas, pois o cuidado envolve não somente a dimensão técnica, mas as dimensões biológicas, psicológicas, sociais e espirituais ${ }^{(2)}$, as quais não serão abordadas neste momento.

Tabela 1- Distribuição dos anunciantes segundo as atividades propostas para a assistência ao idoso referentes a alimentação. Campinas, 1998.

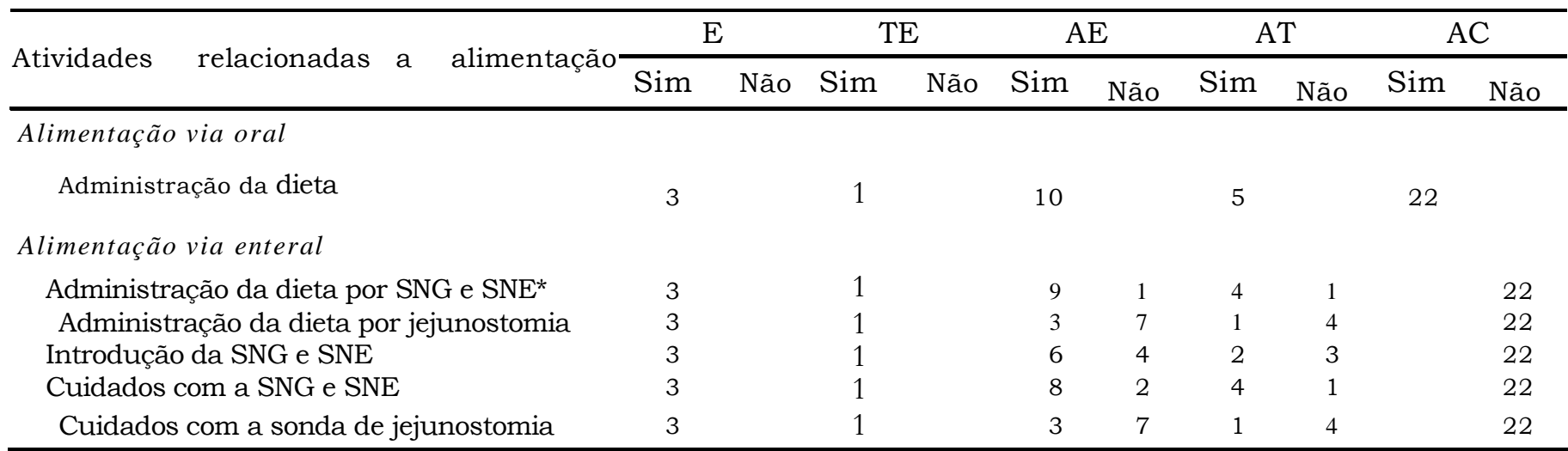

* SNG - Sonda Naso Gástrica

SNE - Sonda Naso Entérica

Os enfermeiros e o técnico de enfermagem se propunham a realizar todos os procedimentos listados, já os acompanhantes referiram não serem capazes de realizar nenhum procedimento relacionado a alimentação, com exceção da administração de dieta via oral.

Apesar de teoricamente capacitados para tal, do total de dez auxiliares de enfermagem entrevistados, um deles não se propunha a administrar a dieta via enteral, quatro deles não realizavam a sondagem e dois se negaram a prestar cuidados com a sonda nasogástrica e nasoenteral. A maior parte deles $(n=7)$ não administrava dieta pela jejunostomia, nem realizava cuidados com a sonda. Cabe destacar que durante a coleta de dados, vários auxiliares de enfermagem demonstraram não ter qualquer conhecimento sobre sondas para alimentação.
Com relação aos atendentes de enfermagem, os cinco entrevistados responderam administrar dieta via oral, quatro deles por sonda e um por jejunostomia; três atendentes afirmaram realizar a introdução da sonda nasogástrica e nasoenteral, enquanto um se propunha a realizar cuidados com as mesmas e quatro com a sonda de jejunostomia.

Embora todos os anunciantes tenham referido administrar a dieta via oral, é importante lembrar que o processo de senescência e senilidade pode levar a dificuldades para a alimentação, como problemas na mastigação (por ausência parcial ou total de dentes) e na deglutição, diminuição dos reflexos com risco de aspiração brônquica do vômito, entre outras. Portanto, o cuidador necessita estar atento ao posicionamento adequado do idoso durante a administração da dieta, assim como quanto a qualidade e quantidade dos alimentos, e sinais e sintomas que requeiram intervenções imediatas. 
Tabela 2 - Distribuição dos anunciantes segundo as atividades propostas para a assistência ao idoso referentes as eliminações. Campinas, 1998.

\begin{tabular}{|c|c|c|c|c|c|c|c|c|c|c|}
\hline \multirow{2}{*}{$\begin{array}{l}\text { Atividades relacionadas } \\
\text { eliminações }\end{array}$} & \multirow{2}{*}{ as } & \multicolumn{2}{|c|}{$\mathrm{E}$} & \multicolumn{3}{|c|}{$\mathrm{AE}$} & \multicolumn{2}{|c|}{ AT } & \multicolumn{2}{|c|}{$\mathrm{AC}$} \\
\hline & & Sim & Não Sim & Não & Sim & Não & Sim & Não & Sim & Não \\
\hline
\end{tabular}

Eliminação por uropen

Instalação do dispositivo

Cuidados com o dispositivo

Cuidados com a higiene

Eliminação por sonda vesical de demora

Passagem e retirada da sonda

Cuidados com a manutenção

Controle da diurese

Cuidados com a higiene

Colocar e retirar a comadre

Eliminação por colostomia

Aplicação e retirada da bolsa e placa

Cuidados com a pele local

Realizar lavagem instestinal

3
3
3

1

1

7

7

3

1

4

4

22

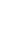

3

1

4

22

22

$\begin{array}{ll}3 & 1 \\ 3 & 1 \\ 3 & 1 \\ 3 & 1 \\ 3 & 1\end{array}$

$\begin{array}{ll}6 & 4 \\ 7 & 3 \\ 8 & 2 \\ 8 & 2 \\ 9 & 1\end{array}$

2
2
2
2
5

$\begin{array}{rrr}3 & 1 & 21 \\ 3 & 1 & 21 \\ 3 & 1 & 21 \\ 3 & 1 & 21 \\ & 13 & 9\end{array}$

\begin{tabular}{llllllll}
3 & 1 & 7 & 3 & 1 & 4 & 2 & 20 \\
3 & 1 & 7 & 3 & 1 & 4 & 2 & 20 \\
3 & 1 & 8 & 2 & 2 & 3 & 5 & 17 \\
\hline
\end{tabular}

Todos os acompanhantes $(n=22)$, referiram não

Os três enfermeiros e o técnico de enfermagem responderam que prestariam todos os cuidados relacionados com as eliminações listados na Tabela 2.

Entre os dez auxiliares, sete afirmaram realizar a instalação e os cuidados necessários no uso do uropen. Já com relação às eliminações pela sonda vesical de demora, seis referiram instalar e retirar a sonda, sete realizavam os cuidados com a manutenção, oito controlavam a diurese e cuidavam da higiene local. Nove dos dez auxiliares de enfermagem mencionaram realizar a colocação e retirada da comadre, porém não foi possivel avaliar se esses auxiliares sabem os cuidados que envolvem esse procedimento quando o paciente é um idoso. Sete deles afirmaram prestar assistência ao idoso colostomizado, como aplicar e retirar a placa e a bolsa de colostomia e prestar os cuidados especiais com a pele local. Oito auxiliares afirmaram realizar lavagem intestinal como relatado por uma das entrevistadas que realizava este procedimento em parente e sentia-se capacitada para repetir em outros idosos. Embora teoricamente, estes profissionais apresentem uma formação específica que pode favorecer sua conscientização quanto aos riscos e peculiaridades no cuidado ao idoso, observamos por meio dos dados obtidos, que nenhuma atividade foi respondida de modo afirmativo pelos dez auxiliares.

Já entre os cinco atendentes de enfermagem, quatro deles responderam que prestam assistência ao idoso com uropen e com bolsa de colostomia; três não se propunham a prestar assistência ao idoso com eliminação por sonda vesical de demora e nem a realizar lavagem intestinal, porém todos afirmaram oferecer cuidados relacionados ao uso da comadre.

oferecer cuidados ao idoso com uropen, demonstrando não terem qualquer conhecimento sobre o dispositivo, enquanto 13 se dispunham a colocar e retirar a comadre. Por outro lado, um, cinco e dois acompanhantes, afirmaram, respectivamente realizar atividades mais complexas relacionadas a sonda vesical de demora, lavagem intestinal e cuidados com a colostomia.

Conforme apontado nos resultados referentes à alimentação, para as atividades relacionadas às eliminações, os atendentes de enfermagem e os acompanhantes sentem-se aptos a realizar cuidados específicos de enfermagem, como sondagem vesical e lavagem intestinal, e demais procedimentos sem orientação de um profissional responsável ou curso específico de formação, colocando em risco a saúde do idoso, além de incorrer numa atividade ilegal. Uma vez não dispensado o cuidado adequado, desde os procedimentos mais simples, até aqueles mais invasivos, podem oferecer riscos para o desenvolvimento de infecções, além de causar lesões na pele fragilizada do idoso e outros danos a sua saúde.

Ademais, sobre as atividades apresentadas até o momento é importante interrogar a capacidade destas pessoas para detectar intercorrências e atuar em situações de emergência com o idoso, principalmente no domicílio. A assistência não se limita somente a realização das atividades propostas, mas envolve todo um planejamento e avaliação constantes, frente as alterações e necessidades do idoso, com intervenções que previnam o surgimento de incapacidades, deformidades e outros comprometimentos da sua saúde. 
Tabela 3 - Distribuição dos anunciantes segundo as atividades propostas para a assistência ao idoso relacionadas à medicação e tratamento. Campinas, 1998.

\begin{tabular}{|c|c|c|c|c|c|c|c|c|c|c|c|c|}
\hline \multirow{2}{*}{$\begin{array}{l}\text { Atividades } \\
\text { medicação }\end{array}$} & \multirow{2}{*}{$\begin{array}{l}\text { relacionadas à } \\
\text { e tratamento }\end{array}$} & \multicolumn{3}{|c|}{$\mathrm{E}$} & \multicolumn{2}{|c|}{$\mathrm{TE}$} & \multicolumn{2}{|c|}{$\mathrm{AE}$} & \multicolumn{2}{|c|}{ AT } & \multicolumn{2}{|c|}{$\mathrm{AC}$} \\
\hline & & Sim & & Não & Sim & Não & Sim & Não & Sim & Não & Sim & Não \\
\hline Bomba de infus & & & 3 & - & 1 & & 5 & 5 & 1 & 4 & & 22 \\
\hline Instalação de $v$ & óclise & & 3 & & 1 & & 8 & 2 & 3 & 2 & 2 & 20 \\
\hline Instalação de o. & yenoterapia & & 3 & & 1 & - & 8 & 2 & 3 & 2 & & 22 \\
\hline \multicolumn{13}{|c|}{ Medicação } \\
\hline Via oral & & & 3 & & 1 & & 10 & & 5 & & 21 & 1 \\
\hline Via intramus & lar & & 3 & & 1 & & 9 & 1 & 5 & & 4 & 18 \\
\hline Via endovenc & & & 3 & & 1 & & 8 & 2 & 1 & 4 & 1 & 21 \\
\hline Via intradérr & & & 3 & & 1 & & 7 & 3 & 1 & 4 & 1 & 21 \\
\hline Via subcutâr & & & & & & & 7 & 3 & 1 & 4 & 1 & 21 \\
\hline Via tópica & & & 3 & & 1 & & 10 & & 5 & & 21 & 1 \\
\hline Via nasal, oc & ar e auricular & & 3 & & 1 & & 10 & & 5 & & 21 & 1 \\
\hline Aplicação de bo & a quente e gelo & & 3 & - & 1 & & 10 & & 5 & & 20 & 2 \\
\hline Inalação & & & 3 & & 1 & & 10 & & 4 & 1 & 11 & 11 \\
\hline \multicolumn{13}{|c|}{ Controle dos sinais vitais } \\
\hline Pressão arte & & & 3 & & 1 & & 10 & & 5 & & 9 & 13 \\
\hline Freqüência & díaca & & 3 & & 1 & & 10 & - & 5 & & 8 & 14 \\
\hline Temperatura & & & 3 & & 1 & & 10 & & 5 & & 14 & 8 \\
\hline Freqüência $r$ & piratória & & 3 & & 1 & & 10 & & 5 & & 8 & 14 \\
\hline
\end{tabular}

Os três enfermeiros e o técnico de enfermagem se propunham a realizar todas atividades relacionadas a medicação e tratamento.

Quanto aos auxiliares de enfermagem, a maioria respondeu administrar a medicação sob as diferentes vias, e os dez afirmaram aplicar bolsa de água quente e de gelo, realizar inalação e os controles de sinais vitais; oito auxiliares instalavam venóclise e oxigenoterapia e cinco referiram administrar medicação pela bomba de infusão.

Entre os atendentes de enfermagem, ocorreram respostas afirmativas mesmo para procedimentos de maior dificuldade e mais invasivos, como manuseio de bomba de infusão $(n=1)$, instalação de venóclise $(n=3)$ e medicações via parenteral: subcutânea $(n=1)$, intradérmica $(n=1)$, intramuscular $(n=5)$, endovenosa $(n=1)$. As demais atividades, que também requerem conhecimento específico, foram: a inalação, proposta por cinco atendentes, aplicação de bolsa de água quente e de gelo, e controle dos sinais vitais por cinco deles.

Já os acompanhantes, não manipulavam a bomba de infusão, porém se dispunham a instalar venóclise $(n=2)$, administrar medicação pelas vias intramuscular $(n=4)$, endovenosa $(n=1)$, intradérmica $(\mathrm{n}=1)$, subcutânea $(\mathrm{n}=1)$, tópica $(\mathrm{n}=21) \mathrm{e}$, nasal, ocular e auricular $(n=21)$.

Se considerarmos que o enfermeiro, o técnico e o auxiliar de enfermagem tenham o preparo técnico-científico para a realização das atividades investigadas, mesmo que não voltado para as peculiaridades do idoso, verificamos que nem todos os auxiliares se propõem a realizá-las. Assim, numa situação hipotética em que eventualmente seja necessária a realização de procedimentos não propostos por esta categoria, a família do idoso terá que contar com outro profissional para tal, o que poderia trazer transtornos para a familia, de natureza fisica, emocional e financeira. Se o auxiliar contasse com a supervisão do enfermeiro, poderia solicitar seu auxilio, prevenindo desse modo fatores de risco para a saúde do idoso e transtornos para a família.

Por outro lado, os dados referentes as atividades propostas pelos atendentes de enfermagem e acompanhantes, revelam a situação crítica de risco a que os idosos estão expostos. Embora alguns procedimentos possam, a principio, serem considerados simples, como por exemplo a aplicação de bolsa de água quente e de gelo, na realidade requerem cuidados específicos na assistência ao idoso frente as alterações da sensibilidade e termorregulação.

Outrossim, não podemos deixar de lembrar do grave risco relacionado à administração de medicamentos (especialmente por via intramuscular e endovenosa), pelos atendentes de enfermagem e acompanhantes, os quais sem qualquer preparo, expõem os idosos a complicações vasculares, nervosas, entre tantas outras relacionadas ao uso de polifármacos, aos aspectos farmacocinéticos e fármaco dinâmicos. 
Tabela 4 - Distribuição dos anunciantes segundo as atividades propostas para a assistência ao idoso relacionadas a integridade cutânea, higiene, locomoção e movimentação. Campinas, 1998.

\begin{tabular}{|c|c|c|c|c|c|c|c|c|c|c|}
\hline \multirow{2}{*}{$\begin{array}{l}\text { Atividades relacionadas a integridade } \\
\text { cutânea, higiene, locomoção e } \\
\text { movimentação }\end{array}$} & \multicolumn{2}{|l|}{$\mathrm{E}$} & \multicolumn{2}{|c|}{$\mathrm{TE}$} & \multicolumn{2}{|c|}{$\mathrm{AE}$} & \multicolumn{2}{|c|}{ AT } & \multicolumn{2}{|c|}{$\mathrm{AC}$} \\
\hline & Sim & Não & Sim & Não & Sim & Não & Sim & Não & Sim & Não \\
\hline \multicolumn{11}{|l|}{ Integridade cutânea } \\
\hline Curativos & 3 & & 1 & & 10 & & 5 & & 12 & 10 \\
\hline Mudança de decúbito & 3 & & 1 & & 10 & & 5 & & 15 & 7 \\
\hline Massagem de conforto & 3 & & 1 & & 10 & & 5 & & 13 & 9 \\
\hline Hidratar e lubrificar da pele & 3 & & 1 & & 10 & & 5 & & 15 & 7 \\
\hline \multicolumn{11}{|l|}{ Higiene } \\
\hline Banho de leito & 3 & & 1 & & 10 & & 5 & & 15 & 7 \\
\hline Banho de aspersão & 3 & & 1 & & 10 & & 5 & & 19 & 3 \\
\hline \multicolumn{11}{|l|}{ Locomoção e movimentação } \\
\hline Transferência & 3 & & 1 & & 10 & & 5 & & 16 & 6 \\
\hline
\end{tabular}

Conforme pode ser verificado na Tabela 4, todos os entrevistados, com exceção dos acompanhantes, responderam realizar procedimentos relacionados a integridade cutânea, higiene, locomoção e movimentação. Entre os 22 acompanhantes, a maioria afirmou realizar as atividades elencadas: curativos $(\mathrm{n}=12)$, mudança de decúbito $(\mathrm{n}=15)$, massagem de conforto $(n=13)$, hidratação e lubrificação da pele $(n=15)$, banho de leito $(n=15)$, banho de aspersão $(n=19)$ e transferência $(n=16)$.

Quanto a integridade cutânea, vale destacar o curativo, entendido como um cuidado essencialmente da enfermagem, o qual exige um conhecimento técnico-científico específico, principalmente perante as novas tecnologias relacionadas ao tratamento de lesões. Assim, requer uma avaliação criteriosa para que a intervenção seja efetiva e eficaz. As demais atividades, mudança de decúbito, massagem de conforto, e hidratação e lubrificação da pele não são menos importantes, principalmente na assistência ao idoso fragilizado ou acamado, na prevenção de deformidades, lesões na pele e outros danos.

A realização da higiene corporal pode ter conseqüências importantes quando não planejado adequadamente, com base na avaliação do idoso, como quedas e fraturas, e lesões na pele. Para tanto, é necessário um preparo do cuidador para poder oferecer uma assistência adequada e especializada, sendo também necessária a supervisão de um profissional com formação específica que possa orientar o cuidado a ser dispensado.

\section{Atividades instrumentais da vida diária}

Conforme descrito anteriormente, as AIVD fazem parte do cotidiano das pessoas e exigem melhor desempenho funcional quando comparadas as atividades da vida diária, portanto, entre os idosos fragilizados, são as primeiras a serem comprometidas.

Temos observado por meio da nossa prática profissional com idosos, que quando a família resolve contratar um cuidador, na maioria das situações, a expectativa é que a pessoa contratada atenda as diferentes demandas, tanto aquelas relacionadas ao cuidado propriamente dito, quanto as atividades que oferecem suporte para o dia-a-dia.

Por outro lado, o atual nível de desemprego favorece a oferta de pessoas para a atuação em diferentes âmbitos, independentemente da sua qualificação profissional.

Estas colocações podem ser confirmadas, segundo os dados obtidos e apresentados a seguir.

Tabela 5 - Distribuição dos anunciantes segundo as atividades propostas relacionadas as AIVD. Campinas, 1998.

\begin{tabular}{|c|c|c|c|c|c|c|c|c|c|c|}
\hline \multirow{2}{*}{ Atividades relacionadas as } & \multicolumn{2}{|c|}{$\mathrm{E}$} & \multicolumn{2}{|l|}{$\mathrm{TE}$} & \multicolumn{2}{|c|}{$\mathrm{AE}$} & \multicolumn{2}{|r|}{$\mathrm{AT}$} & \multicolumn{2}{|r|}{$\mathrm{AC}$} \\
\hline & Sim & Não & Sim & Não & Sim & Não & $\operatorname{Sim}$ & Não & Sim & Não \\
\hline Lavar e passar roupas & 1 & 2 & 1 & & 8 & 2 & 5 & & 19 & 3 \\
\hline Preparar as refeições & 1 & 2 & 1 & & 7 & 3 & 5 & & 19 & 3 \\
\hline Limpar a casa & 1 & 2 & & 1 & 7 & 3 & 5 & & 18 & 4 \\
\hline $\begin{array}{l}\text { Atividades extradomiciliares (ir ao banco, } \\
\text { padaria, supermercado, farmácia, etc.) }\end{array}$ & 1 & 2 & 1 & & 8 & 2 & 5 & & 18 & 4 \\
\hline
\end{tabular}


Observamos na Tabela 5, que em todas as categorias ocorreram respostas afirmativas para as atividades propostas relacionadas as AIVD. Vale destacar que mesmo os anunciantes com formação específica, seja em curso de nivel médio ou superior, responderam realizar os afazeres domésticos e extradomiciliares. Entre os três enfermeiros, um referiu realizar todas as atividades; o técnico de enfermagem se recusou a limpar a casa, e dos dez auxiliares de enfermagem, oito afirmaram cuidar das roupas e efetuar as atividades fora de casa.

Com relação aos anunciantes sem formação específica, os cinco atendentes de enfermagem concordaram em realizar todas as AIVD; dos 22 acompanhantes, 19 cuidariam das roupas e do preparo das refeições e 18 realizariam a limpeza da casa e as atividades extradomiciliares. As respostas dos acompanhantes talvez não causem muita surpresa, pois, como pudemos verificar na primeira parte da pesquisa, a maioria encontra-se em idade produtiva (31 a 50 anos) e frente a escassez de trabalho no mercado, as pessoas se dispõem a atuar nos mais diversos setores. Este fato pode ser confirmado quando observamos na seção de classificados dos jornais, o mesmo anunciante procurando "serviço" ora como acompanhante de idoso, ora como babá, ora como empregada doméstica.

\section{CONSIDERAÇÕES FINAIS}

Os resultados do presente estudo não permitem generalizar as atividades que o cuidador formal do idoso realizam, no entanto, fornecem subsídios para a atual discussão referente a sua atuação.

Muitas das atividades propostas são de competência somente dos profissionais de enfermagem, o que sem dúvida alguma, causa extrema preocupação entre os enfermeiros da área gerontológica e geriátrica. Assim, cabe alertar quanto a necessidade de orientar as familias sobre esta questão e desenvolver projetos, na enfermagem,.relacionados a formação de recursos humanos para o cuidado do idoso no domicílio.

\section{REFERÊNCIAS BIBLIOGRÁFICAS}

(1) São Paulo (Estado). Decisão Coren-SP-DIR/010/99. Diário Oficial do Estado, São Paulo (SP), 24 nov. 1999. Seção 1.

(2) Berger L, Mailloux-Poirier D. Pessoas idosas - uma abordagem global: processo de enfermagem por necessidades. Lisboa: Lusodidacta; 1995.

INSTRUMENTO PARA A ENTREVISTA - PARTE II

Atividades propostas para a Assistência ao Idoso

\section{CUIDADOS DE ENFERMAGEM}

A) Com a alimentação:

-Via Oral: Administração da dieta: -

Via Enteral:

ANEXO I

Administração da dieta por SNG e SNE

Administração da dieta por jejunostomia

Introdução da SNG e SNE

Cuidados com a SNG e SNE

Cuidados com a sonda de jejunostomia

$\begin{array}{lr}\text { Sim () } & \text { Não ( ) } \\ \text { Sim () } & \text { Não () } \\ \text { Si m () } & \text { Não () } \\ \text { Sim () } & \text { Não () } \\ \text { Sim () } & \text { Não () } \\ \text { Sim () } & \text { Não () }\end{array}$

B) Com as eliminacões:

-_Por Uropen:

Instalação do dispositivo

Cuidados com o dispositivo

Cuidados com a higiene

- Por sonda vesical de demora:

Instalação e retirada da sonda

Cuidados com a sua manutenção

Controle de diurese

\begin{tabular}{|c|c|}
\hline $\operatorname{Sim}()$ & Não() \\
\hline $\operatorname{Sim}()$ & Não ( ) \\
\hline $\operatorname{Sim}()$ & Não ( ) \\
\hline Sim $O$ & Não \\
\hline Sim () & Não () \\
\hline $\operatorname{Sim}()$ & Não ( ) \\
\hline
\end{tabular}


uidados com a higiene

- Colocar e retirar a comadre

- Por bolsa de colostomia:

Aplicação e retirada da placa e da

bolsa Cuidados com a pele local

- Realização da lavagem intestinal

C) Medicacões e Tratamentos (terapêutica)

-_Bomba de infusão

- Instalação de venóclise

- Instalação de oxigenoterapia

Administração da medicação:

Via oral

Via intramuscular

Via endovenosa

Via intradérmica

Via subcutânea

Via tópica

Outros (nasal, ocular, auricular)

- Realizar controle dos sinais vitais:

Pressão arterial

Freqüência cardíaca

Temperatura

Freqüência respiratória

D) Integridade cutânea

Realizar curativos

- Realizar mudança de decúbito

- Realizar massagem de conforto

- Realizar hidratação e lubrificação da pele

E) Higiene

-_Realizar banho de leito

- Realizar ou ajudar no banho de aspersão

F) Locomoção e movimentação -

Realizar transferência

\section{ATIVIDADES INSTRUMENTAIS DA VIDA DIÁRIA -}

Lavar e passar roupas

- Preparar as refeições

- Limpar a casa

- Realizar atividades extradomiciliares

( ir ao banco, padaria, farmácia, supermercado, etc.)
Sim () Não () Sim ()

Não ()

Sim () Não () Sim () Não ( ) Sim () Não ()

$\begin{array}{ll}\text { Sim () } & \text { Não ( ) } \\ \text { Sim () } & \text { Não ( ) } \\ \text { Sim () } & \text { Não ( ) - } \\ \text { Sim ( ) } & \text { Não ( ) } \\ \text { Sim ( ) } & \text { Não ( ) } \\ \text { Sim ( ) } & \text { Não ( ) } \\ \operatorname{Sim}(\text { ) } & \text { Não ( ) } \\ \operatorname{Sim}(\text { ) } & \text { Não ( ) } \\ \operatorname{Sim}(\text { ) } & \text { Não ( ) } \\ \operatorname{Sim}(\text { ) } & \text { Não ( ) } \\ \operatorname{Sim}(\text { ) } & \text { Não ( ) } \\ \operatorname{Sim}(\text { ) } & \text { Não ( ) } \\ \operatorname{Sim}() & \text { Não ( ) } \\ \operatorname{Sim}(\text { ) } & \text { Não ( ) } \\ \operatorname{Sim}(\text { ) } & \text { Não ( ) }\end{array}$

\begin{tabular}{|c|c|}
\hline $\mathrm{Sim}$ & ã o ( ) \\
\hline $\operatorname{Sim}()$ & Não ( ) \\
\hline $\mathrm{Sim}()$ & Não() \\
\hline Sim & ã o \\
\hline
\end{tabular}

Sim ( ) Não ( )

Sim ( ) Não ( )

S i m () Não ( ) 